\title{
The Mechanical Properties Enhancement of Biodegradable Aminofunctional Starch/Carboxylate Nitrile Butadiene Rubber Latex Films
}

\author{
M.A. Misman ${ }^{1}$ and A.R. Azura ${ }^{1, *}$ \\ ${ }^{1}$ School of Materials and Mineral Resources Engineering, Engineering Campus, 14300, \\ Nibong Tebal, Penang, Malaysia.
}

Corresponding author email:- srazura@usm.my

\begin{abstract}
The Aminofunctional starch/Carboxylated nitrile butadiene rubber (ANS/XNBR) latex films were prepared with control (0), 5, 10, 15, and $20 \mathrm{phr}$ of ANS loadings. The films were subjected to soil burial test for 8 weeks, which recovered every two weeks to quantify the degraded properties. The control (week 0) and biodegraded films were subjected to physical tests (crosslink density, tensile, and tear strength tests) and biodegradation quantification (mass loss retention, and water vapor transmission rates). The optimum physical properties of ANS/XNBR films were subjected to further assessment of its degradation properties which include morphological (optical and scanning electron microscopes (SEM)), Fourier transform infrared (FTIR) analysis, and thermogravimetric analysis (TGA) respectively. Overall, mechanical properties of biodegraded ANS/XNBR latex films decreased as biodegradation period elapses. The biodegradation assessment via water vapor transmission rates and mass loss analyses indicates the high degree of biodegradation rates were observed with higher loading of ANS/XNBR latex films. Morphological analyses via optical microscope shows the transformation of the films surface opacity during biodegradation test. SEM images depicted the microorganism remains on the film surfaces. For FTIR analysis, the most significant changes in the spectras of the films appeared in the region of $790-3000 \mathrm{~cm}^{-1}$. TGA thermograph shows the thermal retention of the materials decreased, as the biodegradation period elapses for optimum ANS/XNBR latex films. From the results obtained, ANS/XNBR latex films shows optimum degradation and mechanical properties at $10 \mathrm{phr}$ filler loading.
\end{abstract}

Keywords: - Aminofunctional starch (ANS), biodegradation, carboxylated nitrile butadiene rubber (XNBR) latex films. 


\section{Introduction}

Carboxylated nitrile butadiene rubber (XNBR) latex is a colloidal dispersion of acrylonitrilebutadiene-carboxylate terpolymer in a water based medium [1]. The latex is used as a substitute material for nitrile butadiene rubber latex in the production of latex-dip articles. The material has the ability to form an ionic crosslinking, providing an alternative rubber curing system with the absence of an accelerator [2]. Accelerator free latex compound or products provides a less potential of encountering type IV hypoallergenic problem which occurs due to the delay reaction of nitrosamine and nitrosatable compounds [3]. Thus, medical devices which made from the XNBR latex provide better hypoallergenic retention.

However, synthetic latex films are difficult to degrade when disposed [4]. This is due to the lack of an organic substance, such as proteins, lipids, or carbohydrates on the surface of rubber particles and its dispersing medium to be consumed by the microorganism. Thus, attempts were made by introducing a bio-filler such as starch into XNBR latex compound. Starch granules consist of polysaccharide macromolecules of branched amylopectin and straight chain of amylose [5]. They are closely packed with a multiple layer of crystal and amorphous structure of its polymeric chain within the starch granules [6]. As the glucopyranosyl group of starch consist of 3 three hydroxyl groups attached to the main chain, the starch tends to form strong hydrogen bonding among their molecules which resulting in the formation of filler agglomeration during XNBR latex compounding [7]. Thus, modification of its functional group was done by grafting with the acrylonitrile monomer to produce aminofunctional starch (ANS). The similar functional group of ANS with XNBR increases the homogeneity of the filler dispersion in the latex compound [8].

Conventionally, for materials which are enriched with bio fillers, two types of degradation may occur during biodegradation test. First, the ability to degrade is due to the interaction of the materials with surrounding environment such as the soil $\mathrm{pH}$, humidity, and thermomechanical induced environment. This type of degradation which takes place on the surface of the materials up to the molecular level refers to the environmental induced degradation.

As for microorganism induced degradation (biodegradation), the process involves an enzymatic reaction which secreted by potential degrading microorganisms that resides on the surface of the polymeric materials [9]. At first, the microorganism will secrete an enzyme which could solubilize the surface of the polymeric materials and convert the polymers into water soluble extract. The extract would then be consumed by the microorganism as their 
source of nutrients [9]. These complex processes actually could be quantified by examining the formation of microorganisms' colony on the surface of polymer substrate and the progress could easily be quantified by observing the behavior of the microorganisms' colony under microscope. However, to further quantify the progress, several techniques also could be employed. In contrast to the environmental induced degradation, biodegradation usually unnoticed at an early stage and could only occur selectively [10].

In previous research, the ability for ANS/XNBR latex films to biodegrade after 8 weeks of soil burial is confirmed by observing the degraded film surface morphology. The formation of microorganism colonies was detected on the $2^{\text {nd }}$ week of biodegradation assessment. The microorganisms' colonies number and size were increased as the biodegradation period elapses. Even though the biodegradability of ANS/XNBR latex is promising, the results also indicate that, mixing the ANS with XNBR latex compound, it decreased the latex films green strength [8]. The reduction is related to the thermoplastic properties of the starch that lower the films modulus contribute to failure at low stress [11]. Apart from that, it is postulated that the reduction of crosslinking formation between rubber macromolecules also contributes towards the properties decrement. Thus, in order to leverage the physical properties decrement with biodegradability of latex films, a study on the optimum loading was conducted to determine the optimum ANS filler loading that could be admixed during latex compounding.

\section{Materials and methods}

\subsection{Preparation of amino functional starch (ANS)}

Sago starch was gelatinized at $80^{\circ} \mathrm{C}$ with sodium hydroxide $(\mathrm{NaOH})$ by heating mantle in a reaction flask together with five round-neck stoppers. Nitrogen gas was purged in and out to provide an inert environment during the reaction. After the starch gelatinized, the paste was cooled down to $60^{\circ} \mathrm{C}$. An initiator reagent (mixture of Ceric (V) ammonium nitrate and 70\% Nitric acid) was added and mixed for 10 minutes. Purified acrylonitrile was added and the paste was stirred for 2 hours at $600 \mathrm{rpm}$. Table 1 indicates the formulations for preparation of amino functional starch. The ANS degree of substitution as reported is 0.464 [8]. 
94 Table 1: The formulations for preparation of amino functional starch

\begin{tabular}{lcc}
\hline Ingredients & Value & Unit \\
\hline Sago starch & 50.0 & $\mathrm{~g}$ \\
$10 \%$ potassium hydroxide & 12.3 & $\mathrm{~g}$ \\
Initiator reagent & 16.5 & $\mathrm{~g}$ \\
Purified acrylonitrile & 3.0 & Mol/AGU* \\
DI Water & 250.0 & $\mathrm{~g}$ \\
\hline$=$ mol per one anhydroglucose unit of starch
\end{tabular}

${ }^{*} \mathrm{~mol} / \mathrm{AGU}=\mathrm{mol}$ per one anhydroglucose unit of starch

\subsection{Compounding and latex film preparation process.}

Prior addition of ANS paste, the $\mathrm{pH}$ was adjusted with aqueous potassium hydroxide $(\mathrm{KOH})$ to the $\mathrm{pH}$ of XNBR latex compound. The ingredient to prepare the ANS/XNBR latex was indicated in Table 2. ANS/XNBR latex compound were prepared with control $(0), 5,10,15$, and $20 \mathrm{phr}$ loading of ANS in XNBR latex compound. XNBR latex was first mixed with $\mathrm{KOH}$ to stabilize the latex and stirred at $270 \mathrm{rpm}$. Subsequently, the others chemical ingredients were added, followed by the addition of ANS paste and the compound was stirred for an hour. The compound was pre - vulcanized for 1 hour at a temperature of $80^{\circ} \mathrm{C}$ followed by maturation process for 24 hours under room temperature.

The latex films were prepared through the coagulant dipping process. Clean aluminium plate was dipped into coagulant (10\% calcium nitrate) for 10 seconds and dried in a convection oven for 15 minutes at a temperature of $100^{\circ} \mathrm{C}$. The plate was taken out and recondition under room temperature until the plate temperature reduced to $50 \mathrm{C}$. The plate was dipped into ANS/XNBR latex compound for 17 seconds and cured in convection oven for 1 hour at a temperature of $100^{\circ} \mathrm{C}$. The ANS/XNBR latex films were designated as ANS/XNBR $x$, where $x=$ loading of phr (e.g. ANS/XNBR 5). 
115 Table 2: Compounding formulation for production of amino functional starch (ANS) / 116 carboxylated nitrile butadiene rubber latex film.

\begin{tabular}{lcc}
\hline Ingredients & Dry weight, phr & Wet weight, g \\
\hline $44.80 \%$ XNBR latex & 100.00 & 223.21 \\
$10.00 \% \mathrm{KOH}$ & 1.00 & 0.10 \\
$42.15 \% \mathrm{MBT}$ & 0.15 & 0.36 \\
$30.80 \% \mathrm{TMTD}$ & 0.15 & 0.49 \\
$55.40 \%$ ZnO & 1.50 & 2.71 \\
$27.65 \%$ ZDEC & 0.70 & 2.53 \\
$26.70 \%$ AnO & 0.50 & 1.87 \\
$36.10 \% \mathrm{~S}$ & 0.50 & 1.39 \\
$10.00 \%$ ANS dispersion & $*$ & $* *$ \\
\hline concentration were varied from 5, 10,15, and 20 phr.
\end{tabular}

117 * The ANS concentration were varied from $5,10,15$, and $20 \mathrm{phr}$.

118 2.3. Biodegradation procedure and testing

119 The biodegradation test was conducted for 8 weeks. The control and ANS/XNBR latex films 120 were divided into 5 sub series according to the week of recovery. All the films were buried in 121 a controlled soil. The soil $\mathrm{pH}$ and moisture were monitored by 3 in 1 soil tester and were kept 122 at $80 \% \mathrm{RH}$ throughout the biodegradation test. The samples were taken out every two weeks. 123 The samples were then leached in distill water at room temperature for 15 minutes and dried 124 in an open air for 18 hours.

\subsection{Swelling test}

126 Small samples of the dried XNBR latex films were cut $(2 \times 2 \mathrm{~cm})$, weighed and placed in 127 glass vials which were filled with chloroform. The vials were stored at $21^{\circ} \mathrm{C}$ for $48 \mathrm{~h}$. The 128 samples were taken out of the solvent, placed in the air for $30 \mathrm{~s}$ to ensure that the solvent was 129 evaporated from the surface and were weighed again. The network density was calculated 130 using Equation 1, 


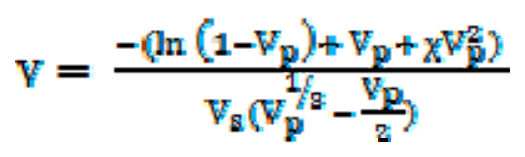

132 Where; $V_{P}$ is the volume fraction of the polymer in the swollen state, $V_{S}$ the molar volume of

133 the solvent, and $\chi$ the Flory-Huggins interaction parameter. The Flory-Huggins interaction 134 parameter was calculated with the following equation, $\chi=0.487+0.228 \times V_{P}$ [12].

The density of the XNBR latex films (polymer) was determined by the hydrostatic weighing method using a Sartorius balance (Goettingen, Germany) with a resolution of $\pm 0.1 \mathrm{mg}$. The apparent weight of the post-cured XNBR films was measured in air and n-heptane and the density was calculated according to Equation 2.

$\rho_{\text {polymer }}=\frac{\left(G_{n}-\text { heptane } \times P_{\text {galr }}\right)}{\left(G_{\text {gir }}-G_{n-h e p t a n e}\right)}$

Where; $\rho_{\text {n-heptane }}$ and $\rho_{\text {air }}$ were the densities of air and n-heptane at $20^{\circ} \mathrm{C} . \mathrm{G}_{\text {air }}$ corresponds to

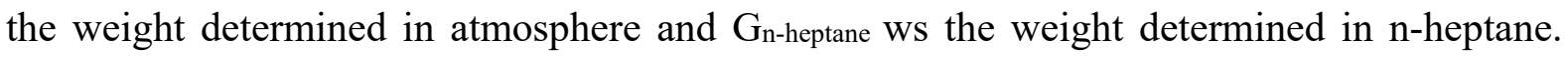
Ten samples were measured and the resulting means value of the density of cross linked XNBR films was $\rho$ XNBR $=1.013 \mathrm{~g} / \mathrm{cm}^{3}$. For biodegraded sample, after the samples had recovered, the samples were washed with distill water before subjected to swelling test.

\subsection{Tensile test}

Tensile test was conducted by using Instron 3366, Machine (Norwood, MA, US) according to ASTM D 412-06. The specimens were cut into dumbbell shape and the thickness of each test piece was recorded. The crosshead speed was set at $600 \mathrm{~mm} / \mathrm{min}$. Five samples were prepared and the average values were calculated. For biodegraded sample, after sample recovery, the samples were washed with distill water and sanitized with ethanol before subjected to tensile test.

\subsection{Tear test}

Tear test was conducted by using Instron 3366 Machine (Norwood, MA, US) according to ASTM D 624-00. The specimens were cut into crescent shape (Die C) and the thickness of each test piece was recorded. The crosshead speed was set at $200 \mathrm{~mm} / \mathrm{min}$. Five samples were prepared and the average values were calculated. For biodegraded sample, after the samples had recovered, the samples were washed with distill water and sanitized with ethanol before subjecting to tear test. 
159

160

161

162

163

164

165

166

167

168

169

170

171

172

173

174

175

176

177

178

179

180

181

182

183

184

185

186

187

188

\subsection{Fourier transform infrared (FTIR) analysis}

FTIR analysis was carried out with Perkin Elmer Spectrum One spectrometer (Shelton, USA), with wave number ranging from 550 to $4000 \mathrm{~cm}^{-1}$ with resolution of $0.5 \mathrm{~cm}^{-1}$. The

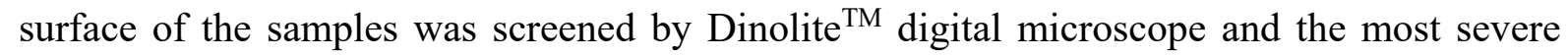
parts were subjected to FTIR analysis.

\subsection{Optical microscope analysis}

The surface of latex films was captured by Meiji digital microscope (San Jose, CA, US) with 10 times magnification. The control and biodegraded samples were washed with distill water and dried in a desiccator to remove any stain or dirt.

\subsection{Scanning electron microscope (SEM) analysis}

SEM analysis was carried out using a Zeiss Supra 35 VP SEM (Oberkochen, Germany) machine with magnification of 5000, and $25000 \mathrm{X}$. The voltage acceleration was set at $5 \mathrm{KV}$. The sample was mounted on aluminium specimen stubs and coated with a plasma ion before analysis. For biodegraded samples, the films were sanitized with chloroform before coating to eliminate foreign substances which may interrupt the coating process. For biodegraded sample, the films were sanitized by ethanol before SEM analysis were conducted. The samples were screened thoroughly by Dinolite TM digital microscope to qualitatively assess the biodegradation process of the films. After the most biodegraded part of the film was determined, the sample was cut into small piece and the desired part was subjected to the SEM analysis.

\subsection{Thermo-gravimetric analysis (TGA)}

Thermo gravimetric analysis (TGA) was carried by Perkin Elmer Pyris 6 (Waltham, USA) machine in a nitrogen environment. $7 \mathrm{mg}$ of sample was transferred into an aluminum pan and placed in the designated sample chamber. The temperature was set from $30^{\circ} \mathrm{C}$ to $600^{\circ} \mathrm{C}$ at heating rates of $10^{\circ} \mathrm{C} / \mathrm{min}$. For biodegraded sample, the samples were washed with distill water and sanitized with ethanol before subjecting to TGA test.

\subsection{Water vapor transmission (WVT) analysis}

WVT analysis was done according to ASTM E96. Biodegraded control and ANS/XNBR latex films were cut into $15 \times 15 \mathrm{~cm}$. A cup was filled with silica gel and ANS/XNBR latex films were used to cover the top of the cup. The cups were then being inserted into 
189 desiccators with a bowl of water at the bottom to ensure a high humidity environment. The 190 desiccator was then sealed to prevent vapor loss except through the test sample. An initial 191 weight was taken and then periodically weighed every day until constant weight was 192 obtained. The increment of weight was monitored until constant weight was achieved. Graph 193 of weight gain against the period of testing was plotted and the best straight line was drawn to 194 obtain the respective gradient. The gradient obtained was divided with the open area of the 195 tested samples. ANS/XNBR latex films WVT rates were calculated by using Equation 3.

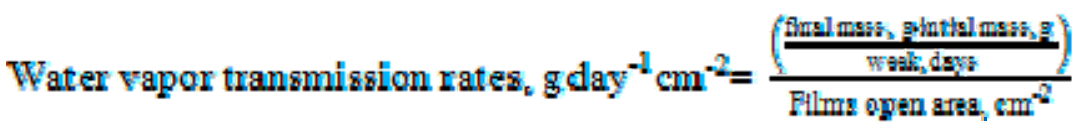

For biodegraded sample, after the samples were recovered, and washed with distill water before the WVT rate measurement.

\subsection{Mass loss analysis}

202

Biodegraded control and ANS/XNBR latex films were taken out every two weeks. The weight of the films was measured after the films were leached with distill water and dried at room temperature for 18 hours. The films mass retention was calculated according to Equation 4.

\section{Results and discussion}

\subsection{Mechanical properties}

\subsubsection{Crosslink density.}

212 From Figure 1 (a), as the biodegradation weeks progressed, the crosslink density of both

213 control and ANS/XNBR latex films reduced with ANS/XNBR latex films undergoes a higher 214 degradation process compared to control films. 
217 Table 3: Percentage of mechanical properties retentions for biodegraded ANS/XNBR latex 218 films.

\begin{tabular}{lrrrr}
\hline Week of recovery & $\mathbf{2}$ & $\mathbf{4}$ & $\mathbf{6}$ & $\mathbf{8}$ \\
\hline (a) Percentage of crosslink density retention, $\%$ & & \\
\hline Control & 87.7 & 28.3 & 17.6 & 13.1 \\
ANS/XNBR 5 & 117 & 57.6 & 49.3 & 32.8 \\
ANS/XNBR 10 & 153.3 & 74.3 & 53.7 & 30 \\
ANS/XNBR 15 & 105.3 & 52.1 & 44.7 & 31.3 \\
ANS/XNBR 20 & - & 60 & 45 & 20.7 \\
\hline
\end{tabular}

(b) Percentage of tensile strength retention, $\%$

\begin{tabular}{lrrrr}
\hline Control & 100 & 94 & 84 & 72 \\
ANS/XNBR 5 & 106 & 93 & 74 & 66 \\
ANS/XNBR 10 & 112 & 109 & 85 & 65 \\
ANS/XNBR 15 & 105 & 79 & 74 & 59 \\
ANS/XNBR 20 & 106 & 81 & 76 & 63 \\
\hline
\end{tabular}

(c) Percentage of elongation at break retention, \%

\begin{tabular}{lcccc}
\hline Control & 99 & 98 & 98 & 94 \\
ANS/XNBR 5 & 95 & 92 & 91 & 86 \\
ANS/XNBR 10 & 94 & 93 & 88 & 87 \\
ANS/XNBR 15 & 87 & 86 & 85 & 81 \\
ANS/XNBR 20 & 96 & 89 & 89 & 85 \\
\hline (d) Percentage of tear strength retention, \% & & & \\
\hline Control & 96 & 81 & 74 & 72 \\
ANS/XNBR 5 & 111 & 80 & 74 & 69 \\
ANS/XNBR 10 & 94 & 75 & 71 & 62 \\
ANS/XNBR 15 & 93 & 71 & 71 & 53 \\
ANS/XNBR 20 & 79 & 56 & 43 & 40
\end{tabular}


223

224

225

226

227

228

229

230

231

232

233

234

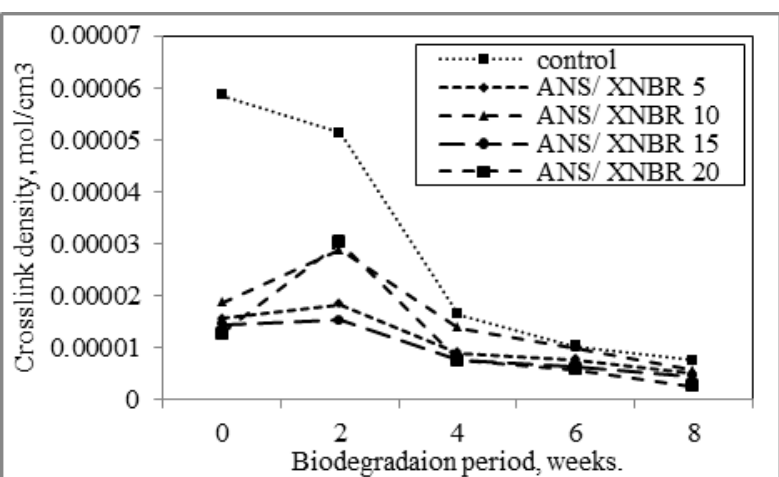

(a)

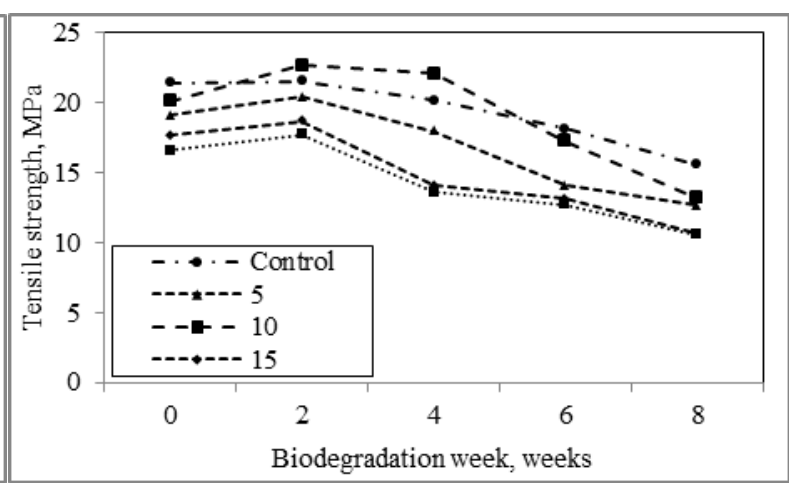

(b)

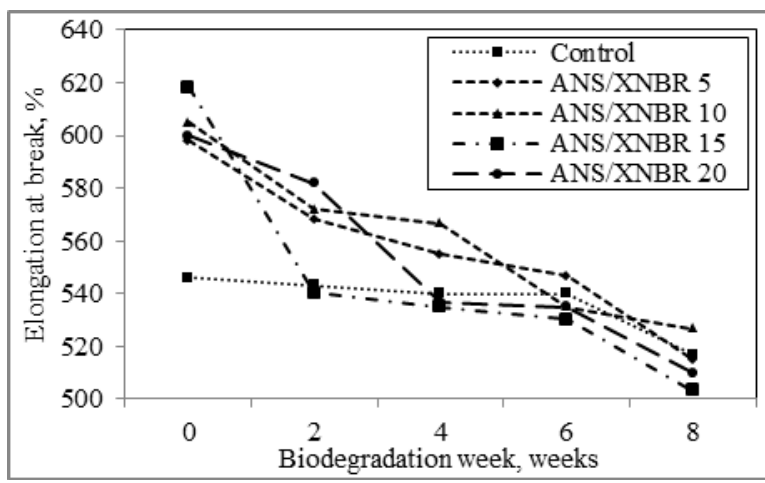

(c)

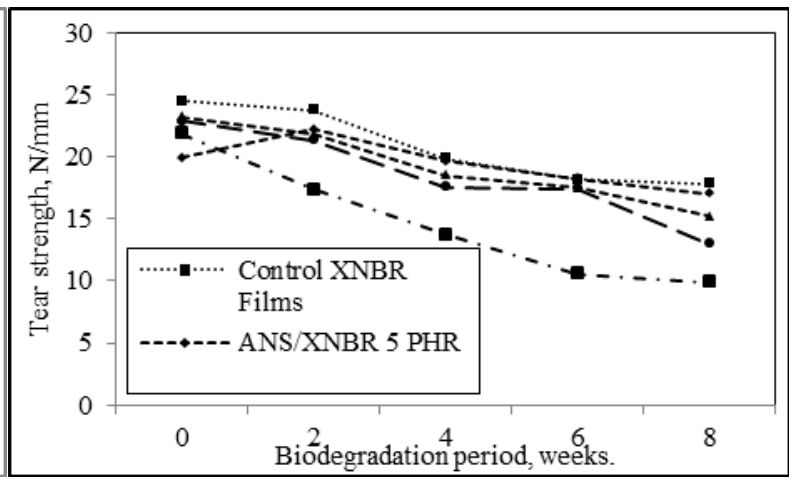

(d)

Figure 1: Mechanical properties of biodegraded ANS/XNBR latex films with respect to their weeks of biodegradation. (a) crosslink density, (b) tensile strength, (c) tear strength, and (d) elongation at break for biodegraded ANS/XNBR latex films.

The addition of ANS into XNBR latex films reduced the crosslink density of the films with ANS/XNBR 20 suffer the lowest. ANS/XNBR 10 shows the highest crosslink density compared to other ANS/XNBR latex films. At the eighth weeks of the biodegradation, the properties decreased for all films.

As indicated in Table 3 (a), for ANS/XNBR latex films, at second week of biodegradation, the films crosslink density increases up to $53 \%$ from its original value. In contrast, for control films, almost $12.3 \%$ of the crosslinks had been decreased. All films show a decreased in the crosslink density at the fourth week of biodegradation process. The highest reduction was observed for ANS/XNBR 10 and 20 with $79 \%$ and $176 \%$ reduction from the previous value recorded indicating rapid biodegradation process compared to other ANS/XNBR films. 
240 For control XNBR latex film, at zero week, the high crosslink density was contributed by the 241 formation of both ionic and sulfur covalent bonding [2]. As the biodegradation process 242 proceeds, the property decreased significantly. The reduction in crosslink density is believed 243 to be due to the solvation of ionic cluster during the biodegradation process $[2,13,14]$. The 244 solvation effect occurred due to the presence of nitrogen element in the compost soil, which 245 was able to solvate the interaction between zinc and oxide ions in the ionic crosslinking 246 system.

247 Meanwhile, the lower crosslink density in ANS/XNBR latex films is due to the occupation of 248 ANS in between rubber macromolecules and hindering the formation of ionic or a sulfur 249 covalent bond. As the biodegradation process progress, the reduction in ANS volume reduced 250 due to microorganism consumption. At second week of the degradation process, due to less hindrance in between adjacent rubber macromolecules in the films, the curing process might occur and further increased the films crosslink density for ANS/XNBR latex films.

253 For control XNBR latex film, during biodegradation period, it is believed that physical degradation might take place dominantly compared to microorganism induced degradation. This is evidenced by the trends shown in Figure 1 (a) that the reduction in crosslink density is proportional to the period of biodegradation. For the ANS/XNBR latex films, the fluctuation in crosslinks density experienced are due to the fluctuation of ANS volume in the films which open the possibility for further crosslinking to occur during the process. This is supported by the fact that the curative agents used in all films are similar as indicated in Table 2.

\subsubsection{Tensile strength.}

From Table 3 (b), in general, the addition of ANS into XNBR latex compound decreased the percentage of tensile retention for ANS/XNBR latex films. It is also indicated that for the second weeks of biodegraded samples, the tensile strength increased with the increased of the crosslink density of the films (Figure 1 (a)). The reduction in tensile strength for the ANS/XNBR latex films was postulated to be due to at least two factors which are; (1) reduction of polymer molecular weight of ANS and XNBR macromolecules, and (2) formation of void/free volume on/in the films.

For the reduction of polymer molecular weight, the process could occur in both ANS and XNBR latex macromolecules. For ANS, the reduction was due to a dextrination process of polysaccharide chain. Such reduction occurred due to the exposure of the films to a certain 
271

272

273

274

275

276

277

278

279

280

281

282

283

284

285

286

287

288

289

290

291

292

293

294

295

296

297

298

299

300

temperature during drying process or due to the enzymatic reaction which induced hydrolysis process in the polysaccharide chain when the sample were buried in the soil [15]. For the dextrination of the polysaccharide chain due to environmental degradation, these phenomena were mainly due to the oxidation process. Similar pathways were postulated to occur in biodegraded samples.

For chain hydrolysis, due to hydrophilicity of ANS and XNBR latex, it could absorb the moisture from the soil to be resided interstitially within the films [16, 17, 18]. These processes could induce an interaction between polysaccharide chains of ANS with water molecules and hence, the hydrolysis of the polysaccharide chain occurred through breakage of the ${ }^{4} \mathrm{C}_{1}$ glucosidic linkages. The phenomenon was best described by Yakubu et al. [19] where reduction in starch enriches sample shown a decreasing trend in its tensile strength as the water uptake increases.

For a chain hydrolysis which were induced by enzymatic reaction, as the water uptakes by the sample increased, the ability of the microorganism to grow and utilize the polysaccharide as their sole source of nutrients. The ability of ANS to be degraded by the microorganism (biodegrade) was well studied by Navarchian et al. and Saroja et al. [20, 21]. Meanwhile, for XNBR latex, the degradation was mainly due to the physical process where the failure of the films was due to the reduction of crosslink density in the films. This was confirmed by the reduction trends in control films despite there was no addition of ANS.

For the reduction of tensile strength due to the increase of free volume/voids in biodegraded samples, the factor was the manifestation of aforementioned mechanisms. These free volume usually formed in a starch rich region and will be occupied by microorganism colony due to high nutrient in the region. As the biodegradation process proceeded, the increased in the size and number of free volume resulting in the film unable to absorb any forces exerted and breaks at lower tensile strength.

\subsubsection{Elongation at break}

From Figure 1 (c), Addition of ANS into XNBR latex compound increased the films elongation at break. However, the elongation at break (EB) for all film decreased with prolonged biodegradation weeks. For control films, even though the EB showed a decreasing trends alongside ANS/XNBR latex films, based on the calculated percentage of EB retention 
301 shown in Table 3 (c), the films with ANS loading has degraded more compared to control

302

303

304

305

306

307

308

309

310

311

312

313

314

315

316

317

318

319

320

321

322

323

324

325

326

327

328

329

330

XNBR latex films.

For control films, the rigidity of the films are the results of a dual crosslinking system. These high density of crosslink network restricts the rubber molecules to deform during applied stress. Thus, even though the film is able to withstand high tensile stress, the films become more rigid. For ANS/XNBR latex films, even though the crosslink is lesser than control films, the ability of the glucopyranosyl ring of starch to deform from a chair to a boat like structure contributes towards the ability of the films to elongate [22].

Table 3 (c) indicates that, as the ANS loading increases, the films undergo a higher reduction in its percent of EB retention. The EB of ANS depends on the flexibility of the ANS segmental chain. Thus, a reduction in its molecular chain has reduced the ability of the polysaccharide molecules to deform with applied stress [23]. Thus, it concludes that the addition of ANS contributes to the ability of the films to elongate upon application of stress.

\subsubsection{Tear strength.}

As shown in Figure 1(d), the tear strength for both control and ANS/XNBR latex films decreased with prolonged weeks of biodegradation. The decreasing property becomes more prominent for ANS/XNBR 20, indicated by the low tear strength retention showed in Table $3(d)$.

For control films, the higher tearing retention at the eighth week of biodegradation experiment signify low degradation rates of tearing strength. This is due to the absence of any hindrance to the formation of ionic or sulfidic linkages during film formation. During the biodegradation process, even though chain fragmentation of XNBR macromolecules occurred, the ability of the films to reinforce before breakage was inevitable by the mechanism of ionic cluster reformation [2].

By comparing with ANS/XNBR latex films, the retention decreases with respect to the weeks of biodegradation. The tear strength of the ANS/XNBR latex films were partly depends on the reinforcing factor of ANS in the films. As the ANS being consumed during biodegradation process, the ability of the films to resist breaking forces was reduced. The phenomenon could be observed in Table 3, where, as the ANS loading increased, the tear strength reduced with ANS/XNBR 20 showed the lowest retention. 
331

332 From Figure 2, as the biodegradation process progress, the percentage of mass loss for 333 ANS/XNBR increased compared to control XNBR latex films. For control XNBR latex 334 films, the increment was not significant at the second week of biodegradation test. As the 335 336 337 338 339 340

341

\subsection{Mass loss analysis.} process continues, the increment becomes significant at fourth, sixth and eighth weeks. The trends were almost similar with ANS/XNBR 10. However, the trends become distinct at fourth to eighth weeks. For 5 and $15 \mathrm{phr}$, the percentage of mass loss was increased constantly with biodegradation duration. The films achieved higher mass loss for $20 \mathrm{phr}$ ANS loading ANS/XNBR films. At these loadings, the percentage of mass loss shown a higher increased only after second weeks of biodegradation process.

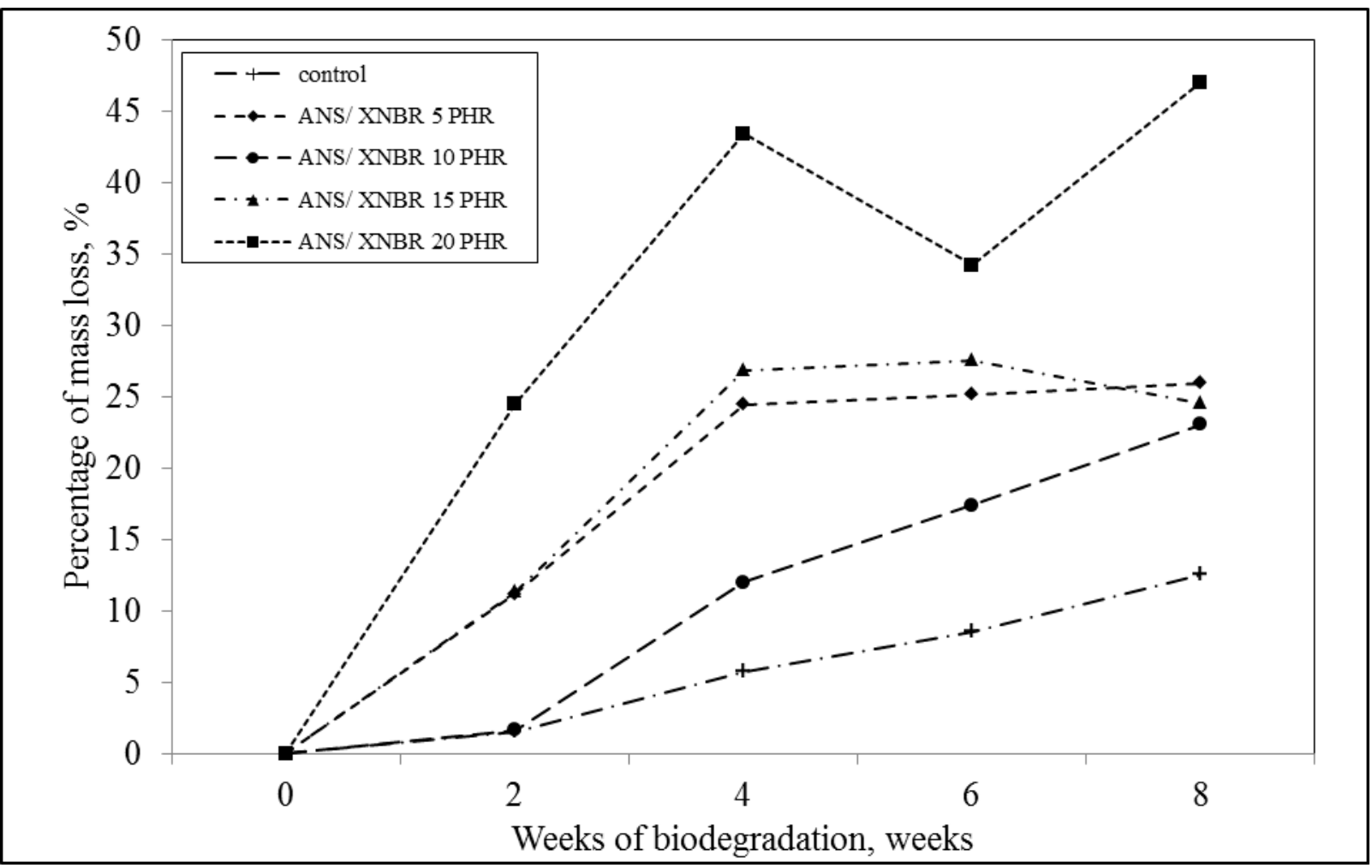

Figure 2: Percentage of mass loss for control and ANS/XNBR latex films with different biodegradation duration.

The presence of ANS in XNBR latex films increased the possibility of the films to biodegrade. Even though at higher filler loading generally did not improved the physical properties of the films. The contribution towards films degradation were more prominent due to the existence of high nutrient in the films as filler loading increased. 
349

350

351

352

353

354

355

356

357

358

359

360

361

362

363

364

365

366

367

\subsection{Water vapor transmission (WVT) analysis.}

In general, the degradation of ANS/XNBR latex films, mechanical properties are related to the loss of film mass which occur through consumption of microorganism. Despite the direct measurement of the film mass retention, the effect also possible to be quantified by determining the water vapor transmission via water vapor transmission rate measurement. In Figure 3, the addition of ANS into XNBR latex compound increased the rates of water vapor transmission through XNBR latex films which increased as the biodegradation weeks progressed. The increased by an average of 1.7 times higher was recorded for ANS/XNBR latex films compared to control XNBR latex films. The highest average increments for all ANS/XNBR latex films WVT was recorded at fourth week, which was 2.19 times higher compared to a control films.

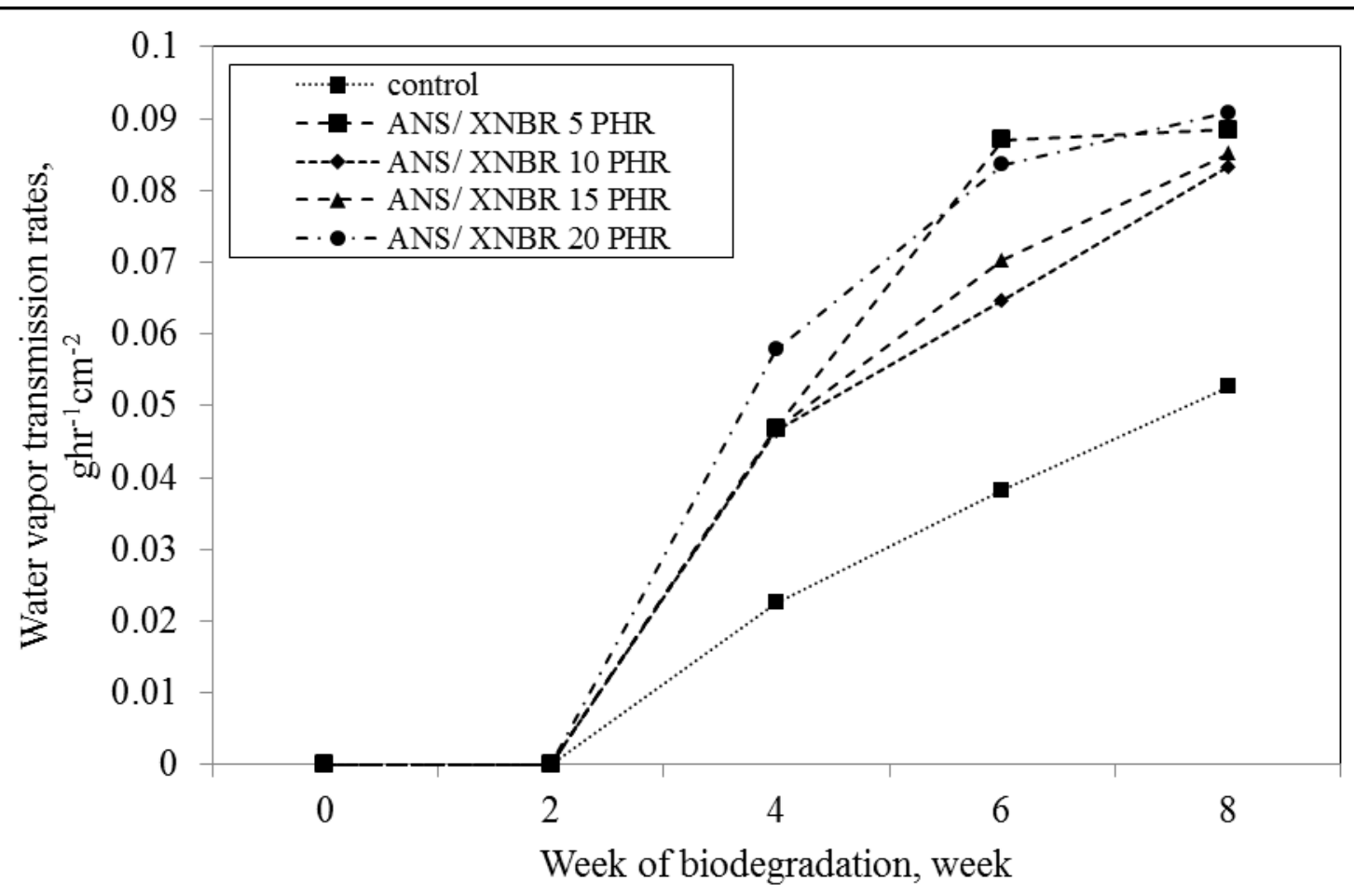

Figure 3: Water vapor transmission (WVT) rates for control and ANS/XNBR latex films with different biodegradation duration.

The formation of voids in ANS/XNBR latex films was vivid as shown in Figure 5. The voids were observed as the manifestation of the microorganism consumption on the latex films. At the end of the biodegradation duration, $20 \mathrm{phr}$ ANS loading shown the highest WVT rates compared to the loading of the ANS/XNBRfilms. Despite higher nutrients consumed by microorganisms that leaves an empty void in the matrix of the ANS/XNBR films, the 
368

369

370

371

372

373

374

375

376

377

378

379

380

381

382

383

384

385

386

387

388

389

390

391

392

393

394

physical of the films itself, which existing void due to irregularities in surface morphology in control weeks, increased the feasibility of the original voids to propagate further during microorganism consumption.

From Figure 3, at the beginning of biodegradation process, the WVT rates were less significant. The trends were tremendously increased at week 2 to week 4 and increasing with slower rates at the sixth week to eighth week. These trends show that the biodegradation process was optimum at second week to sixth week based on the increased of the rates obtained. The rates became slower at sixth to eighth week 8 .

\subsection{Morphological analysis.}

From Figure 4, the appearance of yellowish spot which representing the dextrinated region of the polysaccharide chain in ANS/XNBR latex films becomes significant as the ANS loadings increases and biodegradation weeks progress. For control films, less yellowish spot was detected even in the last week of biodegradation durations. These were contributed from the absence of the polysaccharide region in the films. The degradation path for control films was depending on the degradation of XNBR latex macromolecules and the present of the crosslink in the films. These factors will delay the effect of biodegradation on the films and hence lowering the ability of the films to degrade during soil burial experiments.

For ANS/XNBR, the yellowish region was observed in the first week of the biodegradation experiment. The region continues to grow and become more significant at higher filler loading. These phenomena, which representing the dextrination of starch, indicated that the morphological properties of degraded ANS/XNBR latex films were influenced by the ANS presence in the XNBR films. Further analysis on the surface of the films as presented in SEM images (Figure 5), determine the presence of an irregular circumferences of the void with a lot of internal small pits. Some of the microbial colony remains was still can be observed even after sanitization and coating. 


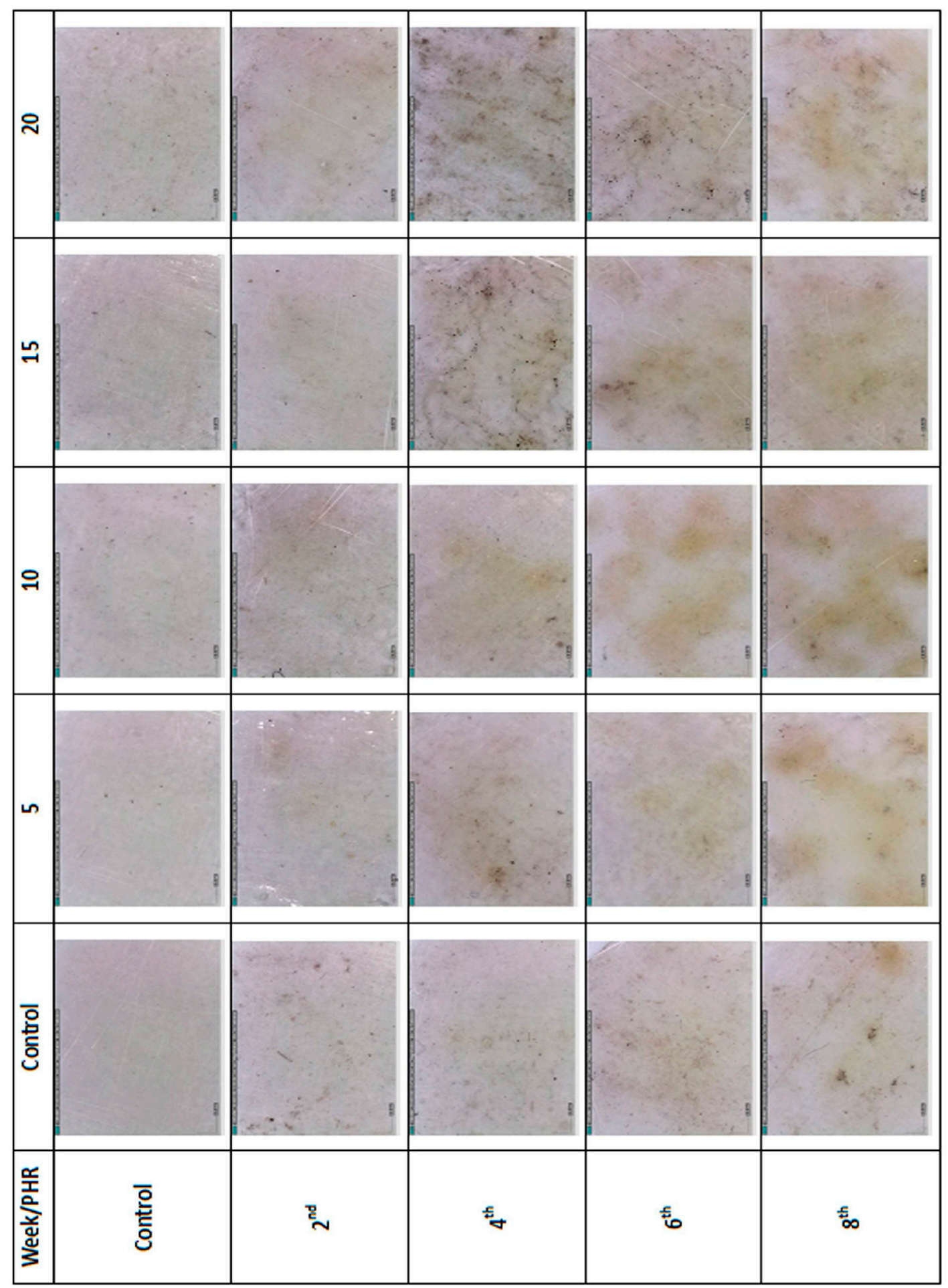

396 Figure 4: The optical analysis of the appearance of control and ANS/XNBR latex films with 397 respect to their filler loading and biodegradation weeks.

398 In order to observe the microorganism colony infections, one sample of ANS/XNBR 10 was 399 selected and observed thoroughly. The films were assessed by optical microscope after the eighth week of soil burial to make an early screening for identification of a specific region to 
401 be focused. SEM analysis was made to rectify the remaining of microorganisms existence in 402 the focused area.

403 From Figure 5 (d), the presence of microorganisms hyphae and mycelia were observed. From 404 the observation of microorganism colony residue shows that the microorganisms hyphae has 405 the ability to penetrate into the films matrix to absorb the nutrients and support the growth of 406 the microorganism colony.

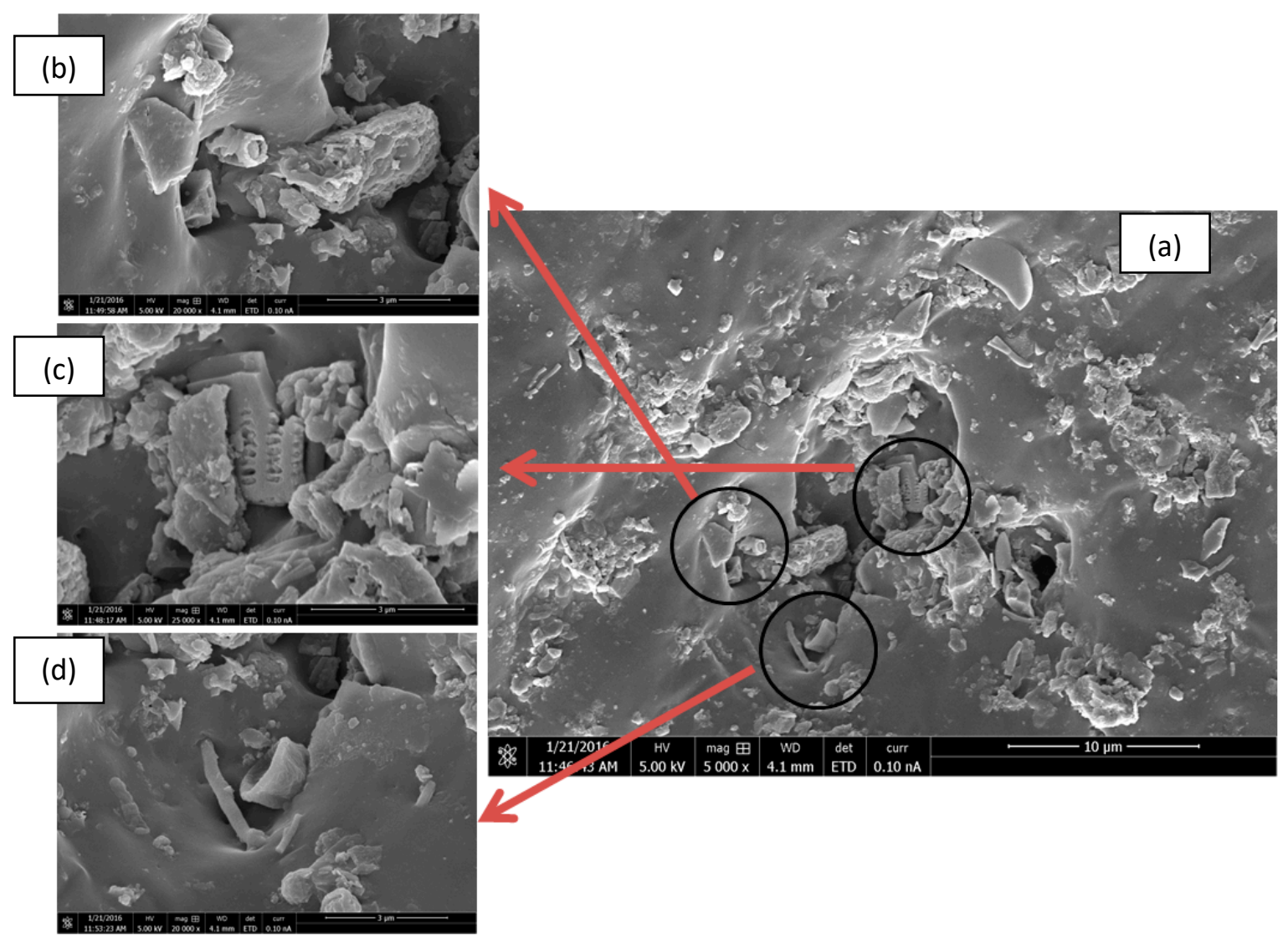

407

408

409

410

411

412

413

414

415

416

417

Figure 5: The morphology of biodegraded ANS/XNBR 10 latex films. (a) Selected area of biodegraded ANS/XNBR latex films, $(b-c)$ the remains of microorganism colony and, (d) a part of the hyphae and mycelia structure of the microorganism embedded in the ANS/XNBR latex films matrix (at magnification of $5000 \mathrm{x}$ for (a), and $20000 \mathrm{x}$ for (b-d)).

\subsection{Fourier Transform infrared (FTIR) analysis}

Figure 6 shows the FTIR spectra of the ANS/XNBR 10 films with respect to its biodegradation weeks. The most significant changes in the spectra of the films appeared in the region of 790-3000 $\mathrm{cm}^{-1}$. To be specific, 6 distinct zones were monitored. For the peaks that correspond to the ANS conformational changes, the peaks are $950 \mathrm{~cm}^{-1}, 1000 \mathrm{~cm}^{-1}$, and $2890 \mathrm{~cm}^{-1}$ which attributed to starch 1,4-glycosidic linkages, the C-O-C bond of 
418 glucopyranose ring and C-H bond of starch aliphatic chain respectively [24-27]. For XNBR 419 conformational changes, peaks at $790 \mathrm{~cm}^{-1}, 1350 \mathrm{~cm}^{-1}$, and $1549 \mathrm{~cm}^{-1}$ were observed. These 420 peaks correspond to the unsaturated $\mathrm{C}=\mathrm{C}$ bond of butadiene in XNBR macromolecules, 421 stretching of $\mathrm{C} \equiv \mathrm{N}$ and bending of $\mathrm{N}-\mathrm{H}$ in a cyano functional group of acrylonitrile 422 respectively [26].

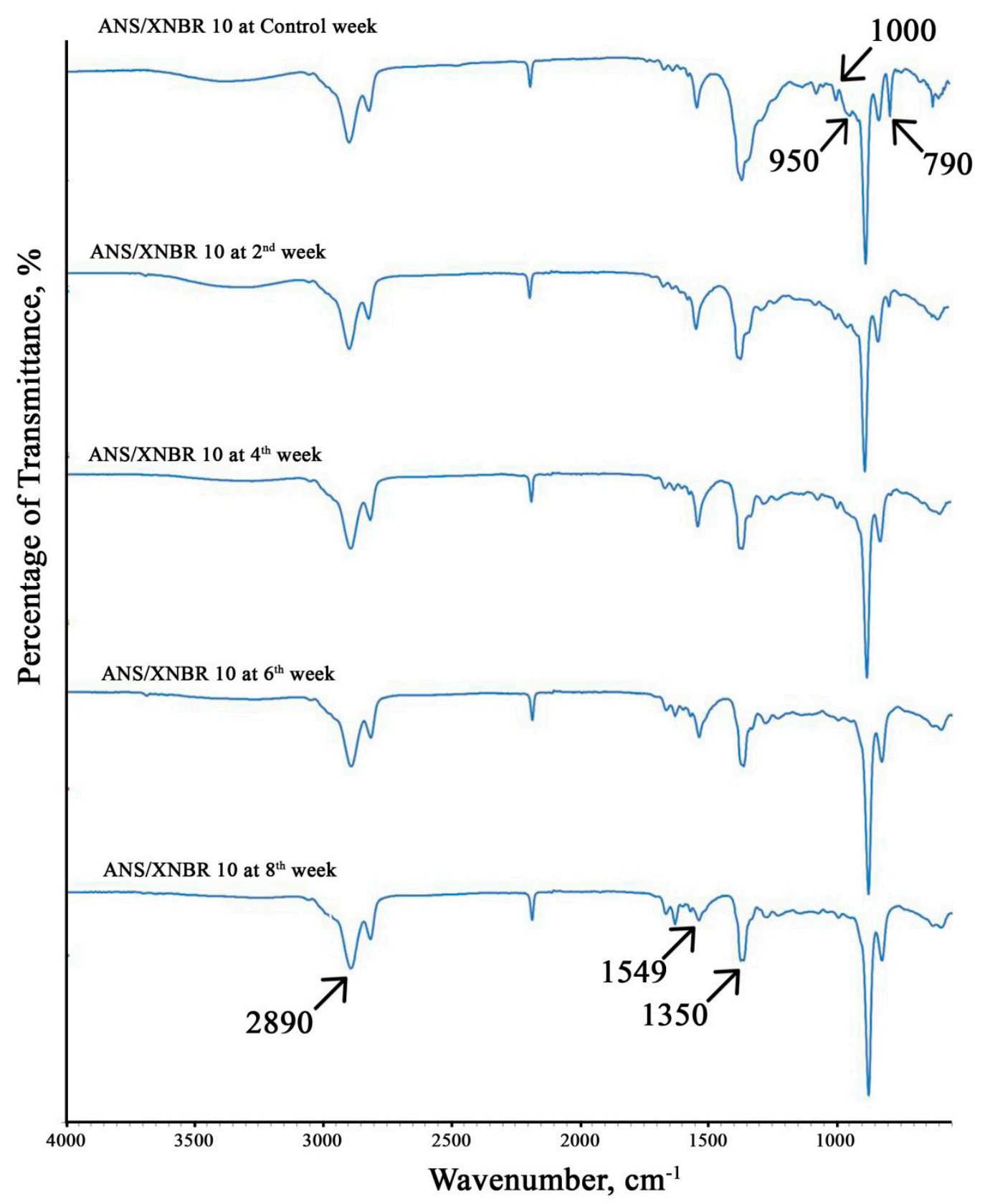

424 Figure 6: FTIR spectra for ANS/XNBR 10 PHR with respect to biodegradation duration.

425 As the biodegradation test was done under aerobic environment, the aerobe microorganism 426 secreted an enzyme which able to depolymerized complex polymer molecules into a smaller 
427 oligomers, dimers, and monomers [28]. The depolymerisation of ANS can be detected by the 428 reduction of $\mathrm{C}-\mathrm{O}-\mathrm{C}$ band with respect to biodegradation weeks. However, insignificant 429 changes were detected for the glycosidic linkages band at peak $950 \mathrm{~cm}^{-1}$ suggesting that the 430 hydrolysis of glucopyranose ring are more dominant compared to the 1,4-glycosidic linkages 431 of polysaccharide. The reduction in $\mathrm{C}-\mathrm{H}$ bond also indicates that the starch aliphatic chain 432 were fragmented and turn into carbon and energy source for the microorganism.

433 For XNBR macromelucules, the process of degradation takes place concurrently with the 434 degradation of ANS macromolecules. The hydrolysis process happens in the unsaturated $\mathrm{C}=\mathrm{C}$ 435 bond of butadiene unit. The scheme, as proposed by Rose and Steinbutchel [29] involved the 436 formation of many potential metabolites. The oxidative cleavage of the unsaturated bond can 437 be identified by observing the reduction of peak intensity at wavenumber $790 \mathrm{~cm}^{-1}$. The 438 depletion of interaction between the ANS and XNBR macromolecules can be detected as the 439 stretching of $\mathrm{C} \equiv \mathrm{N}$ and N-H functional group lessen as characterized in peak $1350 \mathrm{~cm}^{-1}$ and $440 \quad 1549 \mathrm{~cm}^{-1}$.

\subsection{Thermo-gravimetric analysis - Differential thermal analysis (TGA-DTG).}

442 Figure 7 shows the TGA-DTG thermograph for control XNBR latex films at control week 443 until the last week of biodegradation duration. At control weeks, the thermograph shows lots 444 of maximum peaks which indicating that there are lots of materials degraded during the tests. 445 However, the maximum peaks occurred at $550{ }^{\circ} \mathrm{C}$ and the samples are kept degrading even 446 though at maximum pre-set temperature $\left(600{ }^{\circ} \mathrm{C}\right)$. The thermal properties of control XNBR 447 latex films also shown the films undergo at least three times decomposition process where the 448 maximum decomposition was recorded at $550{ }^{\circ} \mathrm{C}$. These observations indicate that the 449 enthalpy changes (endothermic reaction) during thermal decomposition of control XNBR 450 latex films at zero weeks were higher and the films are thermally stable compared to biodegraded control XNBR latex films. 

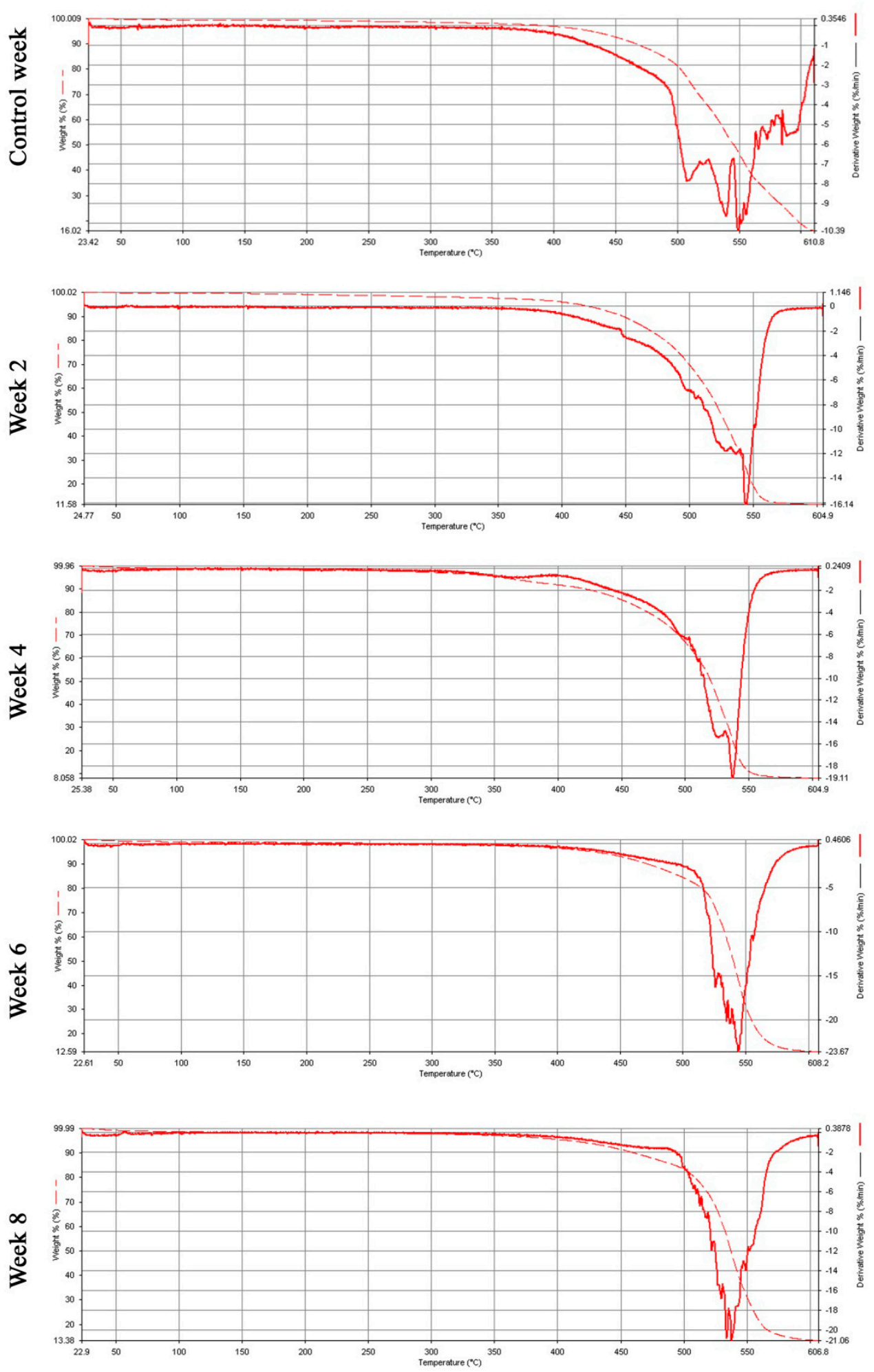

453 Figure 7: TGA-DTG thermograph for control XNBR latex films with different 454 biodegradation duration.

455 As the biodegradation experiment progress, the major peaks become diminished indicating 456 the integrity of the film to withstand high temperature in the thermal degradation process also 
457 decreased. The area under the DTG peaks has also become narrower suggesting that the 458 endothermic reaction of the films was reduced. These were due to less thermal energy are 459 required by the sample to decomposed as biodegradation weeks progress.

460 During the biodegradation process, for control XNBR latex films, a reduction in its 461 crosslinking either ionic or sulfidic will reduce the molecular entanglement in the matrix. The 462 reduction will contribute to the flexibility of the XNBR macromolecules and could deform 463 easily due to the absence of any restriction. Thus, as the thermal energy supply increases, the 464 molecules breaking energy could be achieved, which contributes the easy macromolecules to 465 break. These indicate that the materials will show a rapid transition in its physical properties 466 at high temperature.

467 For ANS/XNBR latex films, as shown in Figure 8, similar trends were recorded. However, at 468 zero week, four physical transitions were recorded which translated into four major peaks on 469 the thermograph. The area under the DTG curve also was larger compared to biodegraded 470 samples. The two early peaks which registered at $\pm 510-540{ }^{\circ} \mathrm{C}$ could be attributed to the 471 degradation of ANS in the films whilst the latter peaks $\left( \pm 540-550{ }^{\circ} \mathrm{C}\right)$ could be attributed to 472 the degradation of XNBR part in the films. It is also concluded that the thermal resistance by 473 the ANS and XNBR part are simultaneously pronounced. These were due to the differences 474 in ANS content in the sample even though the sampling was taken from ANS/XNBR 10 latex 475 films. However, at the last week of biodegradation process, the peaks converged into single 476 peaks and the properties were shifted more towards the XNBR latex degrading temperature. 477 These signified the reduction of ANS content in ANS/XNBR latex films due to microbial 478 consumption. 

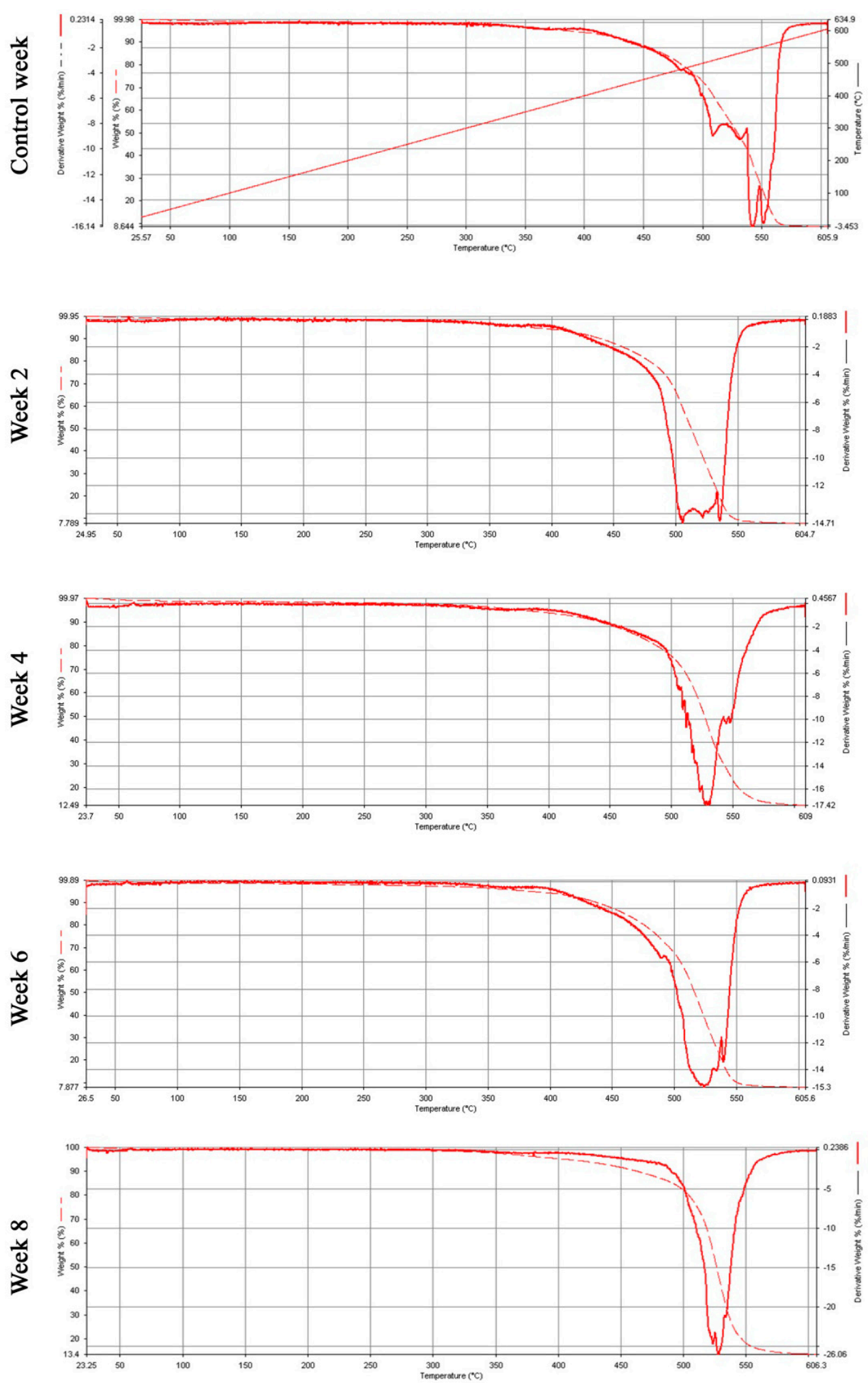
biodegradation duration. 
484

485

486

487

488

489

490

491

492

493

494

495

496

497

498

499

500

501

502

503

504

505

506

507

508

509

510

511

512

513

514

\section{Conclusion}

Addition of ANS into XNBR latex compound reduces the mechanical properties of the composite ANS/XNBR latex films. As the loading increases, further reduction was recorded. This is due to the reduction of crosslink density between rubber macromolecules which was hindered by the formation and increased of the ANS rich region. As the biodegradation process proceeds, the microbial activity in the ANS rich region, which was evidenced in optical and SEM micrograph, further reduces the mechanical property of the latex films. This is due to the scission of the starch aliphatic chain during the biodegradation process that reduce the interaction between ANS and XNBR molecules. The event was evidenced in the FTIR spectral analysis,which indicates that the reductions in starch aliphatic chains at peak $1607 \mathrm{~cm}^{-1}$ and the stretching of N-H bond at peak $3302 \mathrm{~cm}^{-1}$ of the spectrograph. The biodegradation process also leads to increase in the formation of microvoids in ANS/XNBR latex films which was depicted from SEM micrographs and the increased of the WVT rates recorded. This process also was recorded by the TGA thermograph which indicates that as the biodegradation process progressed, the material degradation temperatures reduced.

\section{Acknowledgement}

The Authors would like to thank the School of Materials and Mineral Resources Engineering and USM Postdoctoral fellow scheme 2017 for providing the necessary equipment and financial assistance during the research. Special thanks to MOHE for providing research grants (FRGS grant No. 203 / PBAHAN / 6071353) to finance the research.

\section{References}

[1] Fujii Y., Kawasaki-Shi K., Ikeda A. Highly saturated carboxylated nitrile copolymer rubber and process for the production there of, European patent, 1999, EP $0933381 \mathrm{~A} 1$.

[2] Mandal U.K., \& Tripathy D.K. Dynamic mechanical spectroscopic studies on plasticization of an ionic elastomer based on carboxylated nitrile butadiene rubber by ammonia, Polymer, 1996, 37(25): 5739-5742.

[3] Travas J.S., Jelencic J., Bravar M., Frobe Z. Characterization of the natural rubber vulcanizate obtained by different accelerators, European Polym Journal, 1996, 32(12): 1395-1401.

[4] Tsuchii, A. Microbial degradation of natural rubber, Progress in Industrial Microbiology, 1995, 32: 177-187. 
515 [5] Parker R., Ring S.G. The physical chemistry of Starch. In; Polysaccharide: Structural

516

517

518

519

520

521

522

523

524

525

526

527

528

529

530

531

532

533

534

535

536

537

538

539

540

541

542

543

544

545

546

547

548 Diversity, and Functional Versatility $2^{\text {nd }}$ edn. Marcel Dekker, New York, USA, 2004.

[6] Zeeman S.C., Kossman J., Smith A.M. Starch: Its metabolism, evolution and biotechnological modification in plastics, Annual Review of Plant Biology, 2012, 209234.

[7] Garcia N.L., Fama L., D‘Accorso N.B., Goganes S. Biodegradable starch nanocomposites. In: Vijay KT, Manju KT (Eds) Eco-friendly Polymer nanocomposites: Processing and Properties, Springer, New Delhi, India, 2015.

[8] Misman M.A., Azura A.R., Hamid Z.A.A. The physical and degradation properties of starch-graft-acrylonitrile/ carboxylated nitrile butadiene rubber latex film, Carbohydrate Polymers, 2015, 128: 1 - 10.

[9] Shah A.A., Fariha H., Abdul H., Safia A. Biological degradation of plastics: A comprehensive review, Biotechnology Advances, 2008, 26(3): 246-265.

[10] Yutaka T., Calabia B.P., Charles U.U., Seiichi A. Biodegradability of plastics, International Journal of Molecular Science, 2009, 10(9): 3722-3742.

[11] Melissa G.A.V., Mariana A.D.S., Lucielen O.D.S., Marissa M.B. Natural-based plasticizers and biopolymer films: A review, European Polymer Journal, 2011, 47(3): 254-263.

[12] Lenko D., Sandr S., Armin T., Raimund S., Armin H., Wolfgang K. Dual crosslinking of carboxylated nitrile butadiene rubber latex employing the thiol-ene photoreaction, Journal of Applied Polymer Science, 2013, 129(5): 2735-2743.

[13] Eisenberg A., King M. Ion Containing Polymer: Physical properties and structure, Academic press, New York, 1977.

[14] Eisenberg A. Clustering of Ions in Organic Polymers. A Theoretical Approach, Macromolecules, 1970, 3(2): 147-154.

[15] Bai Y., Liming C., James D., Elliot P.G., Yong-Cheng S. Structural changes from native waxy maize starch granules to cold-water-soluble pyrodextrin during thermal treatment. Journal of agricultural and food chemistry, 2014, 62 (18): 4186-4194.

[16] Apopei D.F., Dinu M.V., Dragan E.S. Graft copolymerization of acrylonitrile onto potatoes starch by ceric ion, Digest journal of nanomaterials and biostructures, 2012 7(2): 707-716.

[17] Stout E.I., Donald T., William M.D. Graft copolymers of starch-polyacrylonitrile prepared by ferrous ion-hydrogen peroxide initiation, Journal of Applied Polymer Sciences, 1977, 21(9): 2565-2573. 
[18] Gugliemelli L.A., Weaver M.O., Russell C.R., Rist C.E. Base-hydrolyzed starch polyacrylonitrile (S-PAN) graft copolymer. S-PAN-1:1, PAN M.W. 794,000, Journal of Applied Polymer Science, 1969, 13: 2007-2017.

[19] Yakubu M.K., Musa M.B., Mukaila J. Degradation and moisture absorption study of potato-starch linear low density polyethylene blend, Nigerian Journal of Basic and Applied Science, 2010, 18: 126-129.

[20] Navarchian A.H., Sharafi A., Kermanshahi R.K. Biodegradation study of starch-graftacrylonitrile copolymer, Journal of Polymers and the Environment, 2013, 21(1): 233244.

[21] Saroja N., Shamala T.R., Tharanathan R.N. Biodegradation of starch-gpolyacrylonitrile, a packaging material, by Bacillus cereus, Process Biochemistry, 2000, 36: $119-125$.

[22] Marszalek P.E., Anders F.O., Yuan-Ping P., Julio M.F. Polysaccharide elasticity governed by chair-boat transitions of the glucopyranose ring, Nature, 1998, 396(6712): $661-664$.

[23] Long Y., Christie G. Microstructure and mechanical properties of oriented thermoplastic starches, Journal of Material Science, 2005, 40: 111-116.

[24] Castano J., Guadarrama-Lezama A.Y., Hernandez J., Colin-Cruz M., Castillo S. Preparation, characterization and antifungal properties of polysaccharidepolysaccharide and polysaccharide-protein films, Journal of Materials Science, 2017, 52: 353-366.

[25] Yuen S.N., Choi S.M., Phillips D.L., Ma C.Y. Raman and FTIR spectroscopic study of carboxymethylated non-starch polysaccharide, Food Chemistry, 2008, 114: 1091-1098.

[26] Litvinov V.M., Prajna P.D. Spectroscopy of rubbers and rubbery materials, Rapra technology limited, United Kingdom, 2002.

[27] Gonera A. Aminofunctional Starch Derivatives: Synthesis, Analysis, and Application, Cuvillier verlag, Germany, 2004.

[28] Gu J.G., Gu J.D. Methods currently used in testing microbiological degradation and deterioration of a wide range of polymeric materials with various degree of degradability: A review, Journal of Polymer and Environment, 2005, 13(1): 65-74.

[29] Rose K., Steinbutchel A. Biodegradation of natural rubber and related compounds: Recent insights into a hardly understood catabolic capability of microorganisms, Applied and Environmental Microbiology, 2005. 71(6): 2803-2812. 